\title{
Increased Prefrontal Activity with Aging Reflects Nonspecific Neural Responses Rather than Compensation
}

\author{
(D)Alexa M. Morcom ${ }^{1}$ and ${ }^{-R i c h a r d ~ N . A . ~ H e n s o n ~}{ }^{2,3}$ \\ ${ }^{1}$ Centre for Cognitive Ageing and Cognitive Epidemiology, University of Edinburgh, Edinburgh EH8 9JZ, United Kingdom, ${ }^{2}$ Medical Research Council \\ Cognition and Brain Sciences Unit, Cambridge CB2 3EB, United Kingdom, and ${ }^{3}$ Cambridge Centre for Ageing and Neuroscience (Cam-CAN), University of \\ Cambridge, Cambridge CB2 3EB, United Kingdom
}

Elevated prefrontal cortex activity is often observed in healthy older adults despite declines in their memory and other cognitive functions. According to one view, this activity reflects a compensatory functional posterior-to-anterior shift, which contributes to maintenance of cognitive performance when posterior cortical function is impaired. Alternatively, the increased prefrontal activity may be less efficient or less specific because of structural and neurochemical changes accompanying aging. These accounts are difficult to distinguish on the basis of average activity levels within brain regions. Instead, we used a novel, model-based multivariate analysis technique applied to two independent fMRI datasets from an adult-lifespan human sample $(N=123$ and $N=115$; approximately half female). Standard analysis replicated the age-related increase in average prefrontal activation, but multivariate tests revealed that this activity did not carry additional information. The results contradict the hypothesis of a compensatory posterior-to-anterior shift. Instead, they suggest that the increased prefrontal activation reflects reduced efficiency or specificity rather than compensation.

Key words: aging; compensation; fMRI; memory; multivariate; prefrontal

\section{Significance Statement}

Functional brain imaging studies have often shown increased activity in prefrontal brain regions in older adults. This has been proposed to reflect a compensatory shift to greater reliance on prefrontal cortex (PFC), helping to maintain cognitive function. Alternatively, activity may become less specific as people age. This is a key question in the neuroscience of aging. In this study, we used novel tests of how different brain regions contribute to long- and short-term memory. We found increased activity in PFC in older adults, but this activity carried less information about memory outcomes than activity in visual regions. These findings are relevant for understanding why cognitive abilities decline with age, suggesting that optimal function depends on successful brain maintenance rather than compensation.

\section{Introduction}

It is well established that healthy aging is associated with a decline in cognitive processes such as memory, but mechanistic explanation of this decline is impeded by difficulties in interpreting the underlying brain changes. fMRI of such cognitive processes shows striking increases, as well as decreases, in brain activity

Received June 20, 2017; revised March 15, 2018; accepted March 21, 2018.

Author contributions: A.M.M. wrote the first draft of the paper; A.M.M. and R.N.A.H. edited the paper; A.M.M. designed research; A.M.M. and R.N.A.H. performed research; A.M.M. and R.N.A.H. analyzed data; A.M.M. and R.N.A.H. wrote the paper.

A.M.M. is a member of the University of Edinburgh Centre for Cognitive Ageing and Cognitive Epidemiology (CCACE), part of the UK cross-council Lifelong Health and Wellbeing Initiative, which is supported by Grant G0700704/84698. The Cambridge Centre for Ageing and Neuroscience (Cam-CAN) research is supported by the Biotechnology and Biological Sciences Research Council (Grant BB/H008217/1). The full Cam-CAN author list can be found here: http://www.cam-can.org/index.php?content= corpauth. R.N.A.H. is funded by the Medical Research Council (Grant SUAG/010 RG91365) with additional support by the European Union's Horizon 2020 Research and Innovation Programme (Grant 732592). We thank Daniel Mitchell for providing univariate models for Experiment 2

Correspondence should be addressed to Alexa M. Morcom, Centre for Cognitive Ageing and Cognitive Epidemiology, University of Edinburgh, 7 George Square, Edinburgh EH8 9JZ, UK. E-mail: alexa.morcom@ed.ac.uk. of older relative to younger adults. One leading theory, the posterior-to-anterior shift in aging (PASA) theory, states that recruitment of anterior regions such as prefrontal cortex (PFC) contributes to maintenance of cognitive performance when posterior cortical function is impaired (Davis et al., 2008; Park and Reuter-Lorenz, 2009; Grady, 2012). Alternatively, age-related increases in PFC activity may reflect reduced efficiency or specificity of neuronal responses, reflecting primarily age-related functional impairment within PFC (West, 1996; Glisky et al., 2001; Park et al., 2004; Raz and Rodrigue, 2006; Nyberg et al., 2012). It is difficult to adjudicate between these theories based on average activity levels within brain regions (Morcom and John-

This is an open-access article distributed under the terms of the Creative Commons Attribution License Creative Commons Attribution 4.0 International, which permits unrestricted use, distribution and reproduction in any medium provided that the original work is properly attributed. 
a

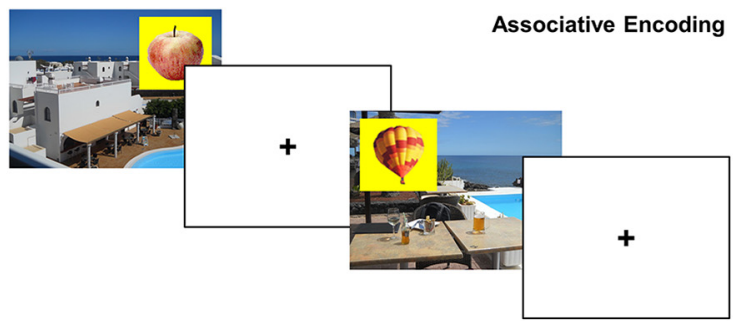

b

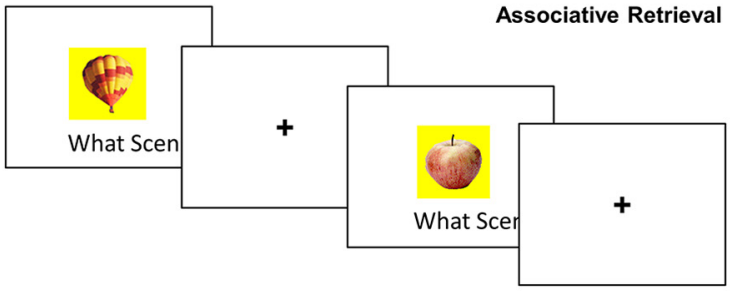

C

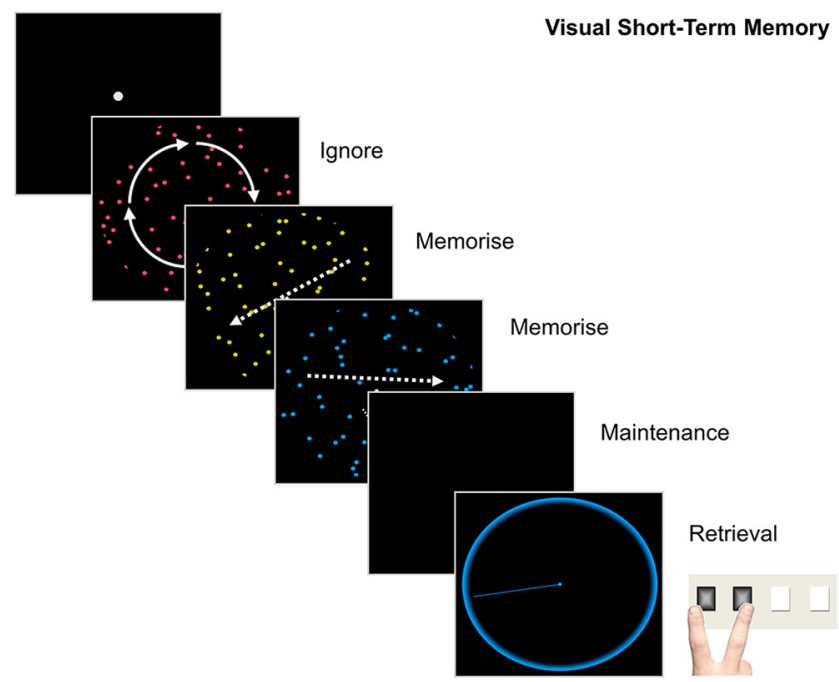

Figure 1. Memory tasks. $\boldsymbol{a}, \boldsymbol{b}$, LTM task. c, STM task. $\boldsymbol{a}$, Associative encoding. In the scanned study phase of the LTM task, participants were asked to make up a story that linked each object with its background scene ( 120 trials total). A scene with positive valence is illustrated. 0 neach trial, the scene was presented for $2 \mathrm{~s}$, the object was superimposed for $7.5 \mathrm{~s}$, and finally the $s c r e e n$ was blanked for $0.5 \mathrm{~s}$ before the next trial. $\boldsymbol{b}$, Associative retrieval. At test (out of the scanner), each object was presented again and, after a measure of priming, item memory, and background valence memory, participants were asked to verbally describe the scene with which it was paired at study. The latter verbal recall was scored as correct or incorrect, which was then used to classify the trials at study into "remembered" and "forgotten" (see text for details). The example illustrates encoding and retrieval of a trial with neutral valence. c, An example trial of STM task with memory load of two items. Trials began with fixation dot for $7 \mathrm{~s}$. On each trial, three dot displays were displayed in red, yellow, and blue for $500 \mathrm{~ms}$ each ( $250 \mathrm{~ms}$ gap). To vary load, the dots in one, two, or three of the dot displays moved in a consistent direction (the to-be-ignored displays rotated). After the last display, the screen was blanked for an $8 \mathrm{~s}$ maintenance period and then the probe display presented a colored circle to indicate which dot display to recall (red, yellow, or blue). Participants had up to $5 \mathrm{~s}$ to adjust the pointer until the direction matched that of the to-be-remembered display.

son, 2015), so we used a novel multivariate approach to directly test predictions of the PASA theory.

With multivariate methods that examine distributed patterns of brain activity over many voxels, one can ask whether increased anterior activity provides additional information and if this information goes beyond that provided by posterior cortical regions. Such increases in the information represented by PFC activity would support theories that attribute additional PFC recruitment to compensatory mechanisms. We used a modelbased decoding approach called multivariate Bayes (MVB) (Friston et al., 2008; Morcom and Friston, 2012; Chadwick et al., 2014), which estimates the patterns of activity that best predict a target cognitive outcome. Importantly, MVB allows formal comparison of models comprising different brain regions such as PFC, posterior cortex, or their combination.

In this study, we applied MVB to fMRI data from two different paradigms in population-derived, adult-lifespan samples $(N=$ 123 and $N=115$, age range $19-88$ years; Shafto et al., 2014). In the first, long-term memory (LTM) experiment, participants were scanned while encoding new memories of unique pairings of objects and background scenes and the target cognitive outcome was whether these associations were subsequently remembered (Fig. 1a). A previous behavioral study in an independent sample showed a strong decline in such associative memory across the adult lifespan (Henson et al., 2016). To test whether findings generalized across tasks and cognitive domains, as PASA predicts (Davis et al., 2008), we replicated our findings in a second, visual short-term memory (STM) experiment. In the STM experiment, a separate sample of participants was scanned while maintaining visual dot patterns online for a few seconds in the presence of distraction and the target cognitive outcome was the increase in the number of patterns to be maintained (i.e., load; Fig. 1b). Increases in PFC activity have frequently been reported in older adults in similar tasks (Grady et al., 1998; Cabeza et al., 2004), although sometimes activity reductions are found at higher loads (Cappell et al., 2010).
We defined two regions of interest (ROIs): posterior visual cortex (PVC), comprising lateral occipital and fusiform cortex, and PFC, comprising the ventrolateral, dorsolateral, superior, and anterior regions (Fig. 2a). These ROIs were based on previous fMRI studies of memory tasks and those cited in the context of the PASA theory (Davis et al., 2008; Maillet and Rajah, 2014).

\section{Materials and Methods}

\section{Experiment 1: LTM encoding}

Participants. A healthy, population-derived adult lifespan human sample $(N=123 ; 19-88$ years; 66 female) was collected as part of the Cambridge Centre for Ageing and Neuroscience (Cam-CAN) study (Shafto et al., 2014). Participants were fluent English speakers in good physical and mental health. Exclusion criteria included a low Mini Mental State Examination (MMSE) score $(\leq 24)$, serious current medical or psychiatric problems or poor hearing or vision, as well as standard MRI safety criteria. Two participants were excluded from fMRI analysis as subsequent memory could not successfully be decoded from either ROI (see "Experimental design and statistical analysis" section). Two further participants were excluded because of statistical outlier values in the analysis of univariate subsequent memory effects (see "Statistics for criteria" section). The experiment used a within-participant design, so all participants received all the task conditions. Therefore, randomization and blinding were not required. The study was approved by the Cambridgeshire 2 (now East of England-Cambridge Central) Research Ethics Committee. Participants gave informed written consent.

Materials. Stimuli were 160 pictures of everyday emotionally neutral objects taken from Smith et al. (2004). For the study phase, objects were presented within a square yellow background on one of 120 scenes from the International Affective Picture System (IAPS) emotional pictures database (Lang et al., 1997). Scenes were grouped into 40 per valence (positive, neutral, or negative), selected based on a pilot study, with the same randomized trial order for each valence condition for all participants. To control for stimulus effects, the 160 objects were divided randomly into four sets and the allocation of object sets to scene valence rotated across participants in four different counterbalances (for further details, see Henson et al., 2016).

Behavioral procedure. The paradigm is summarized in Figure 1a. The scanned study phase comprised 120 trials, presented in two $10 \mathrm{~min}$ 


\section{LTM ENCODING}

\section{a}
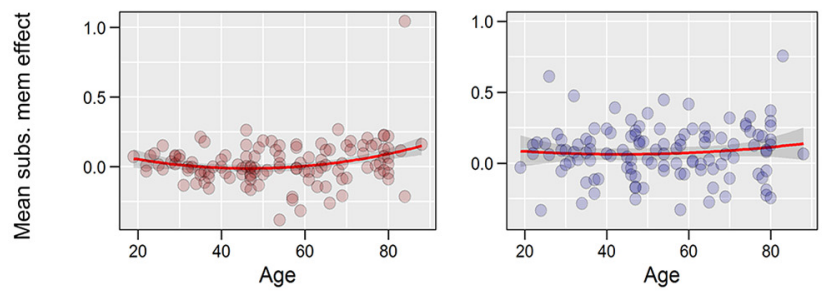

b
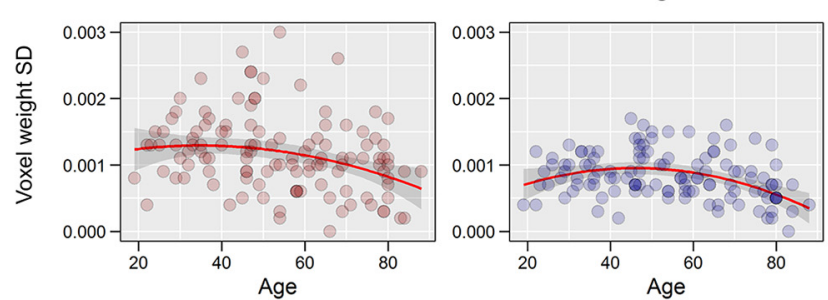

e

Anterior (PFC)

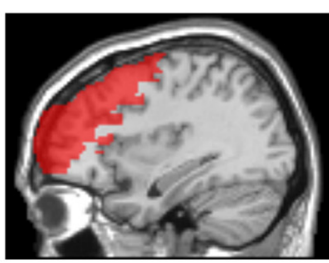

STM MAINTENANCE

C
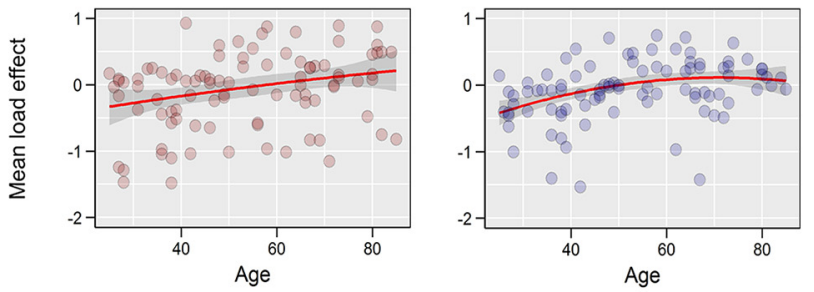

d Standard deviation of multivariate voxel weights in ROI
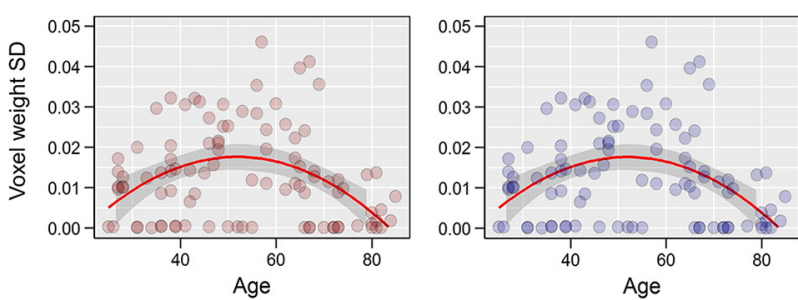

Posterior (PVC)

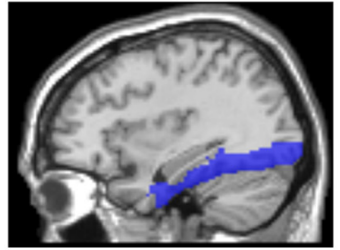

Figure 2. Relationship between age and univariate and multivariate effects within ROls. $\boldsymbol{a}$, Univariate subsequent memory effects (mean activity for remembered - forgotten), showing increased activity with age in PFC but not PVC. $\boldsymbol{b}$, Spread of multivariate responses predicting subsequent memory (SD of fitted MVB voxel weights), showing reduced spread of responses with age in both ROIs. c, Univariate effects of load (positive linear contrast) during STM maintenance, showing increased activity with age in both ROls. $\boldsymbol{e}$, Spread of multivariate responses during STM maintenance predicting increasing load, showing reduced spread of responses with age in both ROls. Red and blue lines are robust-fitted second-order polynomial regression lines and shaded areas show $95 \%$ confidence intervals. $\boldsymbol{e}$, PVC (blue) and PFC (red) ROls overlaid on sagittal section $(x=+36)$ of a canonical T1-weighted brain image. Note that $y$-axis scales are not comparable across tasks.

blocks separated by a short break. On each study trial, a background scene was first presented for $2 \mathrm{~s}$ and an object was then superimposed for $7.5 \mathrm{~s}$ slightly above center and either to the left or right. Participants were asked to create a story that linked the object to the scene and to press a button when they had made the story. To equate the amount of time spent processing the story, participants were asked to continue to elaborate it until the scene and object disappeared. A blank screen of $0.5 \mathrm{~s}$ was then presented before the next trial. Participants were informed that the task would include some pleasant and unpleasant scenes. They were not told that their memory would be tested later. A practice session of six study trials was given just beforehand.

The test phase took place outside the scanner after a short break of $\sim 10$ min involving refreshment and conversation with the experimenter. The 120 objects from the study phase were presented again, randomly intermixed with 40 new objects, and divided into four blocks lasting $\sim 20 \mathrm{~min}$ each. The first stage of each test trial involved tests of priming, item memory, and memory for the picture valence (for details, see Henson et al., 2016). However, for the present fMRI analysis, we focused on the fourth question in each trial. Participants were asked to verbally recall the scene that had been paired with the test object at study. Trials at study in which scenes were correctly recalled at test, in terms of detail or gist, were scored as "remembered." Remaining trials were split according to whether the scenes could not be recalled ("associative misses"), for which an incorrect scene was described instead ("associative intrusions"), or for which the object was not recognized ("item misses"). Initial analyses showed no evidence that valence interacted with age, so trials were collapsed across valence (see "Behavioral results" section). Table 1 summarizes the trial numbers per condition split by age tertile.
Table 1. Trial numbers divided by condition

\begin{tabular}{llll}
\hline & $\begin{array}{l}\text { Younger } \\
(19-45 \text { years }) \\
n=38\end{array}$ & $\begin{array}{l}\text { Middle age } \\
(46-64 \text { years }) \\
n=43\end{array}$ & $\begin{array}{l}\text { Older } \\
\text { (65-88 years) } \\
n=42\end{array}$ \\
\hline $\begin{array}{l}\text { Remembered } \\
\text { Forgotten }\end{array}$ & $55(25)$ & $44(24)$ & $23(15)$ \\
$\quad$ Associative miss & $31(13)$ & $38(17)$ & $46(18)$ \\
Associative intrusion & $11(8)$ & $13(10)$ & $10(8)$ \\
Item miss & $22(15)$ & $25(17)$ & $42(24)$ \\
\hline
\end{tabular}

Remembered refers to trials with correct object recognition and scene recall; associative miss, trials with correct object recognition but no scene recall; associative intrusion, trials with correct object recognition but recall of an incorrect scene; item miss, trials with misclassification of the object as unstudied. Data are split by age tertile and are shown as means (SD).

For the main imaging analyses, we combined the three types of forgotten trial to maximize power. However, in case processes that lead to subsequent memory for associative memory versus item memory differ (Dennis et al., 2008; Mattson et al., 2014), we also ran a subsidiary imaging analyses with item misses excluded.

Imaging data acquisition and preprocessing. The MRI data were collected using a Siemens 3 T TIM TRIO system. MR data preprocessing and univariate analysis were performed with SPM12 software (Wellcome Department of Imaging Neuroscience, London, www.fil.ion.ucl.ac.uk/spm), release 4537, implemented in the AA 4.0 pipeline (https://github.com/ rhodricusack/automaticanalysis). The functional images were acquired using $\mathrm{T} 2{ }^{\star}$-weighted data from a gradient-echo echoplanar imaging (EPI) sequence. A total of 320 volumes were acquired in each of the two study sessions, each containing 32 axial slices (acquired in descending or- 
der), slice thickness of $3.7 \mathrm{~mm}$ with an interslice gap of $20 \%$ (for whole brain coverage including cerebellum; $\mathrm{TR}=1970 \mathrm{~ms}$; $\mathrm{TE}=30$ $\mathrm{ms}$; flip angle $=78$ degrees; FOV $=192 \mathrm{~mm} \times 192 \mathrm{~mm}$; voxel-size $=$ $3 \mathrm{~mm} \times 3 \mathrm{~mm} \times 4.44 \mathrm{~mm}$ ). A structural image was also acquired with a T1-weighted 3D magnetization prepared rapid gradient echo (MPRAGE) sequence $(\mathrm{TR}=2250 \mathrm{~ms}, \mathrm{TE}=2.98 \mathrm{~ms}, \mathrm{TI}=900 \mathrm{~ms}, 190$ $\mathrm{Hz}$ per pixel; flip angle 9 deg; FOV $256 \times 240 \times 192 \mathrm{~mm}$; GRAPPA acceleration factor 2).

The structural images were rigid-body registered with an MNI template brain, bias corrected, segmented, and warped to match a gray matter template created from the whole CamCAN Stage 3 sample $(N=272)$ using DARTEL (Ashburner, 2007; Taylor et al., 2017). This template was subsequently affine transformed to standard Montreal Neurological Institute (MNI) space. The functional images were then spatially realigned, interpolated in time to correct for the different slice acquisition times, rigid-body coregistered to the structural image, transformed to MNI space using the warps and affine transforms from the structural image, and resliced to $3 \times 3 \times 3 \mathrm{~mm}$ voxels.

Univariate imaging analysis. For each participant, a general linear model (GLM) was constructed, comprising three neural components per trial: (1) a delta function at onset of the background scene, (2) an epoch of $7.5 \mathrm{~s}$ that onset with the appearance of the object $(2 \mathrm{~s}$ after onset of scene) and offset when both object and scene disappeared, and (3) a delta function for each keypress. Each neural component was convolved with a canonical hemodynamic response function to create a regressor in the GLM. The scene onset events were split into three types (i.e., three regressors) according to the valence of the scene on each trial, whereas the keypress events were modeled by the same regressor for all trials (together, these four regressors served to model trial-locked responses that were not of interest). The responses of interest were captured by the epoch neural component, during which participants were actively relating the scene and object (see "Behavioral procedure" section). The duration of this component did not depend on response time because participants were instructed to continue to link the object and scene mentally for the full duration of the display.

For the principal GLMs, the epoch component was split into six types (regressors) according to the three scene valences and two types of subsequent memory; that is, study trials for which the scenes were correctly recalled ("remembered") and those for which the scenes could not be recalled, an incorrect scene was described instead, or the object was not recognized ("forgotten"). When comparing remembered and forgotten trials, we averaged across the three valences because: (1) there was no behavioral evidence of an interaction between age and valence on subsequent memory, (2) there was no imaging evidence of an interaction between age and valence on subsequent memory, and (3) there would have been insufficient numbers of each trial-type to examine each valence separately. Therefore, the main target contrast for the univariate and multivariate analyses were remembered versus forgotten trials.

As noted above, given that encoding of associative (source) information versus item information may differ with regard to additional recruitment and (potentially) to compensation (Dennis et al., 2008; Mattson et al., 2014), we ran a subsidiary analysis in which the "forgotten" category excluded item misses. In these GLMs, study trials were modeled using nine regressors according to the three scene valences and three (rather than two) types of subsequent memory: trials on which the object was recognized but the scene forgotten or incorrectly recalled ("association forgotten") and trials on which the object was not recognized ("item forgotten"). Participants for whom one of the sessions did not contain at least one trial of each type were removed, leaving $n=109$ (note this involved removal of more participants in the oldest age tertile: 0 removed aged $19-35,2$ aged $46-64$, and 12 aged $65-88$ years). One remaining outlier $(>5 \mathrm{SD})$ on the univariate measures was removed, giving $n=108$. As reported in the Results section, this subsidiary analysis corroborated the findings of the main analysis.

Six additional regressors representing the three rigid body translations and rotations estimated in the realignment stage were included in each GLM to capture residual movement-related artifacts. Finally, the data were scaled to a grand mean of 100 over all voxels and scans within a session and the model was fitted to the data in each voxel. The autocor- relation of the error was estimated using an $\mathrm{AR}(1)$-plus-white-noise model, together with a set of cosines that functioned to high-pass the model and data to $1 / 128 \mathrm{~Hz}$, fit using restricted maximum likelihood. The estimated error autocorrelation was then used to "prewhiten" the model and data and ordinary least squares used to estimate the model parameters. To compute subsequent memory effects, the parameter estimates for the six epoch components were averaged across the two sessions and the three valences (weighted by number of trials per session/ valence) and contrasted directly as remembered minus forgotten (Morcom et al., 2003; Maillet and Rajah, 2014). Univariate statistical analyses were conducted on the mean subsequent memory effect across all voxels in the MVB analysis in each ROI for each participant (see "Multivariate Bayesian decoding" section).

\section{Experiment 2: Visual STM}

Participants. Participants were a separate subset $(N=115 ; 25-86$ years; 54 female) of those recruited to the Cam-CAN study (see Experiment 1 , "Participants," for details). Nineteen participants were excluded from the current analysis as the contrast of interest could not successfully be decoded from either region of interest (see "Multivariate Bayesian decoding" section). None were excluded because of statistical outlier values on the measures used (see "Experimental design and statistical analysis" section for criteria). The experiment used a within-participant design so all participants received all the task conditions.

Materials. The task was adapted from Emrich et al. (2013). Stimuli were three patches of colored dots, one red, one yellow, and one blue. Dots were 0.26 degrees of visual angle (dva) in diameter at a density of 0.7 per square degree and viewed though a circular aperture of diameter 11 dva. As a manipulation of set size, 1,2 , or 3 of the dot displays moved (at $2 \mathrm{dva} / \mathrm{s}$ ) in a single direction that had to be remembered. The other, distractor, displays rotated around a central axis and were be ignored. On $90 \%$ of trials, the probed movements were in one of three directions $\left(7^{\circ}\right.$, $127^{\circ}$, or $\left.247^{\circ} \mathrm{s}\right)$. Other directions were selected at random to avoid subjects learning the target directions. Order of presentation of the three display colors was randomized trial by trial, as was memory load. Rotation direction alternated across trials of a given load.

Behavioral procedure. Each trial began with a gray fixation dot in the middle of a black screen for $5 \mathrm{~s}$, which then turned white for $2 \mathrm{~s}$. Participants then saw the three dot displays for $500 \mathrm{~ms}$ each, with $250 \mathrm{~ms}$ in between. After the third display, an 8 s blank fixation delay was presented, followed by the probe display. The probe display showed a colored circle to indicate which dot display to recall (red, yellow, or blue) with a pointer. Participants had up to $5 \mathrm{~s}$ to adjust the pointer using 2 buttons until it matched the direction of motion of the remembered target dot display. After responding, a third button cleared the probe display. Participants completed three runs of 30 trials per run ( 10 for each load). The direction of the target, the sequential position of the target, and the set size were counterbalanced within each run and presented in random order. Color and position of target were also counterbalanced using a Greco-Latin square design.

Imaging data acquisition and preprocessing. Imaging data were acquired on the same scanner as Experiment 1. Functional T2*-weighted data were acquired using a multi-echo gradient-echo EPI sequence. Approximately 300 volumes were acquired in each of the three visual shortterm memory (VSTM) task sessions (duration depending on response times). Each volume had 34 axial slices (acquired in descending order), slice thickness of $2.9 \mathrm{~mm}$, with an interslice gap of $20 \%$ (FOV $=224$ $\mathrm{mm} \times 224 \mathrm{~mm}, \mathrm{TR}=2000 \mathrm{~ms} ; \mathrm{TE}=12 \mathrm{~ms}, 25 \mathrm{~ms}, 38 \mathrm{~ms}$; flip angle $=$ 78 degrees; voxel-size $=3.5 \mathrm{~mm} \times 3.5 \mathrm{~mm} \times 3.48 \mathrm{~mm}$ ). Structural image sequences were the same as in Experiment 1. The multiple echoes were combined by computing their average weighted by their estimated $\mathrm{T} 2 *$ contrast. The functional images were spatially realigned and interpolated in time to correct for different slice acquisition times. Spatial normalization used the "new segment" protocol in SPM12 (Ashburner and Friston, 2005). Participants' structural scans were coregistered to their mean functional image and then segmented into six tissue classes. Functional images were rigid-body coregistered to the structural image then transformed to MNI space using the warps and affine transforms estimated from the structural image and resliced to $2 \times 2 \times 2 \mathrm{~mm}$ voxels. 
Univariate imaging analysis. The GLM for each participant comprised three neural components per trial: (1) encoding, modeled as an epoch of $1 \mathrm{~s}$ duration at onset of the first moving dot pattern; (2) maintenance, modeled as an epoch of $4 \mathrm{~s}$ at offset of the last moving dot pattern $(2.25 \mathrm{~s}$ after onset of 1); and (3) probe, a delta function at the time of the participant's response. These components were each split into three types (regressors) according to the three STM load levels. As in Experiment 1, six additional regressors were added, representing the motion parameters estimated in the realignment stage. Finally. the data were scaled to a grand mean of 100 over all voxels and scans within a session. To confirm that this dataset was suitable as a replication of Experiment 1's multivariate results, we first checked that at least one significant cluster within the PFC ROI showed increased univariate activity in older people. This was done using a standard analysis of effects of a linear contrast of increasing VSTM load on activity during the delay period, whole-brain corrected for multiple comparisons at $p<0.05$ (voxel threshold), and a linear contrast of age. Details of this analysis are not reported and it did not contribute to ROI selection, which was the same as for Experiment 1. Note that the continuous nature of the judgment in the VSTM task precludes definition of individual trials as correct or incorrect (rather, performance is used to estimate continuous summary measures for each participant, as in Emrich et al., 2013). Therefore, all trials were included in the fMRI analysis and the main target contrast for the univariate and multivariate analyses was the linear effect of load from 1 to 3 during the delay period.

ROIs

ROIs were defined using WFU PickAtlas (http://fmri.wfubmc.edu/, version 3.0.5) with AAL and Talairach atlases (Lancaster et al., 2000; Tzourio-Mazoyer et al., 2002; Maldjian et al., 2003). The PVC mask comprised bilateral lateral occipital cortex and fusiform cortex (from AAL, fusiform, and middle occipital gyri) and the PFC mask comprised bilateral ventrolateral, dorsolateral, superior, and anterior regions: from $\mathrm{AAL}$, the inferior frontal gyrus (IFG; both pars triangularis and pars orbitalis), lateral part of the middle frontal gyrus (MFG), superior frontal gyrus (SFG), and from Talairach, Brodmann area 10 (BA10), dilation factor $=1$. In the subregion analyses, separate masks were created for BA10, IFG, MFG, and SFG (regions included in the BA10 mask were excluded from the other masks).

\section{Multivariate Bayesian decoding}

A series of MVB decoding models were fit to assess the information about subsequent memory carried by individual ROIs or combinations of ROIs. Each MVB decoding model is based on the same design matrix of experimental variables used in the univariate GLM, but the mapping is reversed: many physiological data features (derived from fMRI activity in multiple voxels) are used to predict a psychological target variable (Friston et al., 2008). This target (outcome) variable is specified as a contrast. In Experiment 1 (LTM) the outcome was subsequent memory and, in Experiment 2 (STM), it was the linear increase in STM load during maintenance periods. Modeled confounds in the design (all covariates apart from those involved in the target contrast) are removed from both target and predictor variables.

Each MVB model is fit using hierarchal parametric empirical Bayes, specifying empirical priors on the data features (voxelwise activity) in terms of patterns over voxel features and the variances of the pattern weights. Because decoding models operating on multiple voxels (relative to scans) are ill posed, these spatial priors on the patterns of voxel weights act as constraints in the second level of the hierarchical model. MVB also uses an overall sparsity (hyper) prior in pattern space that embodies the expectation that a few patterns make a substantial contribution to the decoding and most make a small contribution. The pattern weights specifying the mapping of data features to the target variable are optimized with a greedy search algorithm using a standard variational scheme which iterates until the optimum set size is reached (Friston et al., 2007). This is done by maximizing the free energy, which provides an upper bound on the Bayesian log evidence (the marginal probability of the data given that model). The evidence for different models predicting the same psychological variable can then be compared by computing the differ- ence in their log evidences, giving the log (marginal) likelihood ratio test (Bayes factor) (Friston et al., 2007; Chadwick et al., 2012; Morcom and Friston, 2012). In this work, the main outcome measures were the log evidence for each model and the set of fitted weights for all patterns (voxels) in the ROI, which can be examined to assess their distribution over voxels and the contributions of different combinations of voxels. These analyses were implemented in SPM12 v6486 and custom MATLAB scripts.

We used a sparse spatial prior, in which each pattern is an individual voxel (Morcom and Friston, 2012; Chadwick et al., 2014; Hulme et al., 2014; Maass et al., 2014). Features (voxels) for MVB analysis were selected using an orthogonal contrast and a leave-one-participant-out scheme. For each participant and ROI, these were the 1000 voxels with the strongest responses to the task: in Experiment 1 (LTM), the six epoch regressors modeling object onsets in the GLM and, in Experiment 2 (STM), the three epoch regressors modeling maintenance periods in the GLM (defined using an F contrast in all other participants testing variance explained by these regressors, regardless of valence, or subsequent memory). We first checked that the target memory variables could reliably be decoded from the selected features by contrasting the evidence for each model with the evidence for models in which the design matrix (and therefore the target variable) had been randomly phase shuffled, taking the mean over 20 repetitions and comparing log evidence for real versus phase-shuffled models. One-tailed $t$ tests compared the difference in real versus shuffled model evidences to a hypothesized population mean difference of 3, which would reflect good Bayesian evidence for the real over the shuffled models. These showed that the difference in log evidence was robustly greater than 3 in both PVC $\left(t_{(118)}=6.08, p<0.0001\right)$, the unbiased estimate of the amount of variance explained in the population $\left[\left(r_{\text {adj }}^{2}\right)=\right.$ 0.225 , mean difference $=9.72$; and PFC, $t_{(118)}=7.70, p<0.0001, r_{\mathrm{adj}}^{2}=$ 0.323 , mean difference $=11.8]$. The same applied to Experiment 2 : for PVC, $t_{(95)}=8.42, p<0.0001, r_{\text {adj }}^{2}=0.415$, mean difference $=23.0$, and PFC, $t_{(95)}=11.4, p<0.0001, r_{\text {adj }}^{2}=0.569$, mean difference $=35.0$. To confirm that the sparse prior represented the best spatial model, we then compared the log evidence with that for models with smooth spatial priors, in which each pattern is a local weighted mean of voxels (Gaussian FWHM $=8$ ). For Experiment 1 (LTM): log evidence was substantially greater for the sparse priors in both ROIs: in PVC, $t_{(118)}=18.4, p<0.0001, r_{\text {adj }}^{2}=0.737$, and PFC, $t_{(118)}=18.0, r_{\text {adj }}^{2}=0.728, p<0.0001$, two-tailed tests. The same was true for Experiment 2 (STM): for PVC, $t_{(95)}=10.3, r_{\text {adj }}^{2}=0.464, p<0.0001$, and PFC, $t_{(95)}=14.9, r_{\text {adj }}^{2}=0.650, p<0.0001$.

Unlike univariate activation measures such as subsequent memory effects, but like other pattern information methods, MVB finds the best nondirectional model of activity predicting the target variable, so positive and negative pattern weights are equally important. Therefore, the principle MVB measure of interest for each ROI was the spread (SD) of the weights over voxels, reflecting the degree to which multiple voxels carried substantial information about subsequent memory. To test whether PFC activity was compensatory, we also constructed a novel measure of the contribution of PFC to subsequent memory. This used Bayesian model comparison within participants to assess whether a joint PVC-PFC model boosted prediction of subsequent memory relative to a PVC-only model. The PASA hypothesis, in which PFC is engaged to a greater degree in older age and this contributes to cognitive outcomes, predicts that a boost will be more often observed with increasing age. The initial dependent measure was the log model evidence coded categorically for each participant to indicate the outcome of the model comparison. The 3 possible outcomes were as follows: a boost to model evidence for PVC-PFC relative to PVC models (i.e., better prediction of subsequent memory: difference in log evidence $>3$ ), equivalent evidence for the two models $(-3<$ difference in $\log$ evidence $<3$ ), or a reduction in prediction of subsequent memory for PFC-PVC relative to PVC (i.e., difference in log evidence $<-3$ ).

Last, given that the relative contribution of anterior versus posterior regions could change with age, even if the absolute amount of pattern information decreased with age in both regions, we computed a second novel measure: we estimated the PFC contribution to cognitive outcome in terms of the proportion of top-weighted voxels in the joint PVC-PFC model that were located in PFC, as opposed to PVC, derived from joint 
Table 2. Age effects on mean univariate SM effects and spread of multivariate SM effects in the LTM task

\begin{tabular}{|c|c|c|c|c|c|c|c|c|}
\hline \multirow[b]{2}{*}{$\mathrm{ROl} /$ measure } & \multicolumn{2}{|l|}{ Model } & \multicolumn{3}{|l|}{ Linear } & \multicolumn{3}{|l|}{ Quadratic } \\
\hline & $F$ & $p$ & $t$ & $r_{\text {adj }}^{2}$ & $p$ & $T$ & $r_{\text {adj }}^{2}$ & $p$ \\
\hline \multicolumn{9}{|c|}{ Mean univariate SM activation } \\
\hline PVC & 0.426 & 0.654 & 0.728 & - & 0.480 & 0.703 & - & 0.495 \\
\hline PFC-PVC & 0.837 & 0.436 & 0.883 & - & 0.388 & 1.084 & - & 0.293 \\
\hline \multicolumn{9}{|c|}{ Multivariate spread (SD) of SM activity } \\
\hline PFC & 6.36 & 0.00240 & -3.33 & 0.0701 & 0.000998 & -1.44 & - & 0.151 \\
\hline
\end{tabular}

PFC-PVC refers to analyses in which the dependent variable was the difference in each measure between PFC and PVC. $n=119$.

PVC-PFC models. In each participant, the voxels making the strongest contribution to the cognitive outcome, defined as those with absolute voxel weight values $>2$ SDs from the mean, were split according to their anterior versus posterior location. The dependent measure was the proportion of these top voxels located in PFC.

Experimental design and statistical analysis

Sample size was determined by the initial considerations of Stage 3 of the CamCAN study (Shafto et al., 2014 for details). For the LTM experiment, a sensitivity analysis indicated that with $N=123$, we would have $80 \%$ power to detect a small to medium effect explaining $6.5 \%$ of the variance on a two tailed test for a model with 2 predictors $\left(r^{2}=0.0658\right)$. For the STM experiment with $N=115$, the corresponding minimal effect size for $80 \%$ power was $6.9 \%$ of the variance $\left(r^{2}=0.0694\right)$. In our previous report of aging and successful memory encoding, an a priori test of a between-region difference in subsequent memory effects according to age showed a large effect $\left(r^{2}=0.257\right)$ (Morcom et al., 2003).

Age effects on continuous multivariate or univariate dependent measures were tested using robust second-order polynomial regression with "rlm" in the package MASS for R (Venables and Ripley, 2002; MASS version 7.3-45; $\mathrm{R}$ version 3.3.1) with standardized linear and quadratic age predictors. For analysis of covariance for behavioral data we used JASP version 0.8.3.1; JASP Team, 2018. Analysis of outcomes of the between-region MVB model comparison (PVC and PFC combined vs PVC; see Fig. 2 and main text) used ordinal regression with "polr" in MASS. Distributions were also trimmed to remove extreme outliers ( $>5$ $\mathrm{SD}$ above or below the mean). In Experiment 1 (LTM), the two participants (aged 72 and 80 ) with outlier values for univariate effects were also removed from the MVB analyses so the samples examined were comparable. We excluded two further participants (aged 68 and 83) in whom subsequent memory could not be decoded from at least one of the two ROIs (log model evidence $\leq 3$ ), giving $n=119$. In Experiment 2 (STM), we excluded 19 participants in whom VSTM load could not be decoded during maintenance, giving $n=96$. All tests were two-tailed and used an $\alpha$ level of 0.05 .

Where it was important to test for evidence for the null hypothesis over an alternative hypothesis, we supplemented null-hypothesis significance tests with Bayes factors (Wagenmakers, 2007; Rouder et al., 2009). The Bayes factors were estimated using Dienes' online calculator (Dienes, 2014) that operationalizes directional hypotheses such as PASA in terms of a half-normal distribution. Here, we assumed an effect size of 1 SD and therefore defined the half-normal distribution with mean $=0$ and $\mathrm{SD}=$ 1. All statistics and $p$-values are reported to 3 significant figures, except where $p<0.0001$.

\section{Results}

\section{Experiment 1: LTM encoding}

Behavioral results

We examined age effects on the number of trials in each remembered and forgotten condition (Table 2). For remembered trials, there was a significant linear decrease with age $\left(t_{(118)}=-7.30\right.$, $\left.p<0.0001, r_{\text {adj }}^{2}=0.299\right)$ with no significant quadratic component $\left(t_{(118)}=-0.104, p=0.917 ; \alpha=0.0125\right)$. As a consequence, the number of forgotten trials increased with age and this was true for both associative misses (linear $t_{(118)}=4.82, p<0.0001$, $r_{\text {adj }}^{2}=0.150$; quadratic, $\left.t_{(118)}=0.630, p=0.532\right)$ and item misses (linear $t_{(118)}=5.43, p<0.0001, r_{\text {adj }}^{2}=0.186$; quadratic, $t_{(118)}=$ $1.57, p=0.120$ ), although not for associative intrusions (linear $t_{(118)}=-1.38, p=0.163$; quadratic, $\left.t_{(118)}=-2.29, p=0.0221\right)$. Analysis of covariance with the factor of valence (positive, neutral, or negative) showed no interaction between age and valence on the number of remembered items (for linear effect of age, $F_{(2,231)}<1, p=0.486$; quadratic, $\left.F_{(2,231)}=1.59, p=0.206\right)$.

\section{Testing compensation}

Standard univariate activation analyses assessed mean activity in each ROI across all voxels included in the multivariate analysis (see Materials and Methods). Consistent with the PASA account, the increase in activity associated with subsequent memory became more pronounced with age, particularly in later years (linear effect of age, $t_{(118)}=2.43, p=0.0166$; quadratic effect of age, $t_{(118)}=2.58, p=0.0111$; Fig. 2a, Table 2). Age effects in PVC were not significant (Table 2). The age effects in PFC were also present after removal of the older participant with the largest SM effect (although they did not meet our criterion for an outlier; Fig. $2 a$; linear $t_{(117)}=2.14, p=$; quadratic $\left.F(117)=2.31, p=0.033\right)$. In both ROIs, results were very similar for the models excluding forgotten trials for which the items themselves were forgotten (see Materials and Methods; PFC: linear $t_{(107)}=2.22, p=0.0316$; quadratic $t_{(107)}=2.91, p=0.00527$; PVC: linear, $t_{(107)}=1.10$, $p=0.288$; quadratic, $\left.t_{(107)}=1.24, p=0.233\right)$.

If the increasing PFC activation with age reflected compensation, then we would expect the multivariate analyses to show that this increased activity carried additional information about subsequent memory. However, the data revealed a different pattern. In MVB models, like other linear models with multiple predictors, each voxel within an ROI has a weight that captures the unique information that it contributes (in this case, for predicting subsequent memory). Because both positive and negative weights carry information, we summarized the MVB results by the spread (SD) of weights over voxels (see Materials and Methods).

In both ROIs, this spread was markedly reduced during later life $\left(\right.$ PVC: linear $t_{(118)}=-3.49, p=0.000650$; quadratic $t_{(118)}=$ $-3.50, p=0.000621$; PFC: linear $t_{(118)}=-3.33, p=0.000998$; quadratic $t_{(118)}=-1.44, p=0.151$; Figure $2 b$, Table 2). This means that, contrary to a compensatory PASA shift, PFC showed fewer, rather than more, voxels with large positive or negative weights with increasing age. Again, the results were similar for the subsidiary models excluding item misses (PVC: linear $t_{(107)}=$ $-1.41, p=0.158$; quadratic $t_{(107)}=-2.81, p=0.000544$; PFC: linear $t_{(107)}=-2.21, p=0.0280$; quadratic $t_{(107)}=-0.566, p=$ $0.570)$. In contrast, the spread of univariate activities across voxels increased with age in both ROIs (for PVC, linear effect of age, 
a

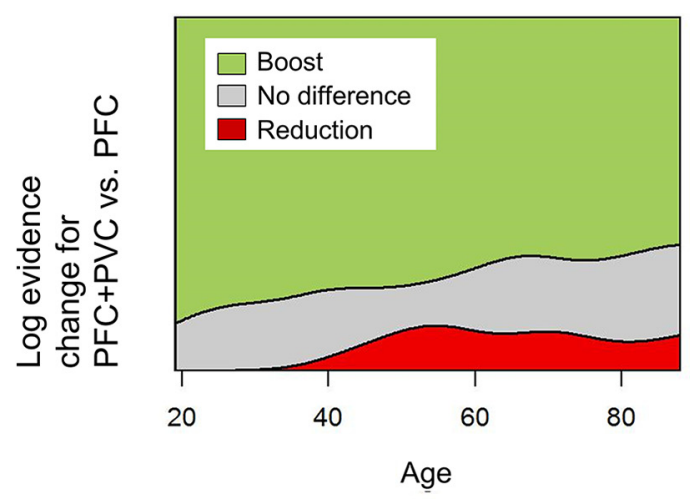

b

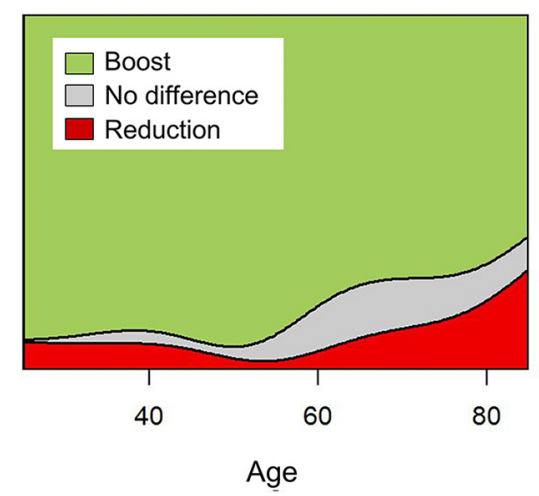

Figure 3. Evidence against a compensatory posterior-to-anterior shift from MVB comparisons between ROIs. Ordinal regression of Bayesian model comparison of combined PVC $+\mathrm{PFC}$ model versus PVC-only model using age to predict outcomes of model comparison: adding PFC to the model boosts prediction of the cognitive outcome (difference in log evidence $>3$ ) or there is no boost $(-3<$ difference $<3)$, or a reduction in log evidence (difference $<-3)$. $\boldsymbol{a}$, LTM. For subsequent memory effects, a boost was no more frequent with increasing age. $\boldsymbol{b}$, STM. For load effects, a boost was less frequent with increasing age.

Table 3. Age effects for PFC subregions in the LTM task

\begin{tabular}{|c|c|c|c|c|c|c|c|c|}
\hline \multirow[b]{2}{*}{ ROI/ measure } & \multicolumn{2}{|c|}{ Model } & \multicolumn{3}{|l|}{ Linear } & \multicolumn{3}{|l|}{ Quadratic } \\
\hline & $F$ & $p$ & $t$ & $r_{\text {adj }}^{2}$ & $p$ & $t$ & $r_{\text {adj }}^{2}$ & $p$ \\
\hline \multicolumn{9}{|l|}{ BA10 } \\
\hline MVB spread (SD) & 8.28 & 0.000433 & -3.75 & 0.0911 & 0.0003249 & -1.86 & - & 0.0623 \\
\hline PFC boost & - & - & -1.44 & - & & -0.321 & - & \\
\hline \multicolumn{9}{|l|}{ IFG } \\
\hline Mean univariate SM & 5.39 & 0.00572 & 2.48 & 0.204 & 0.0152 & 2.72 & 0.227 & 0.00824 \\
\hline \multicolumn{9}{|l|}{ MFG } \\
\hline Mean univariate SM & 1.34 & 0.266 & 1.56 & - & 0.119 & 1.38 & - & 0.169 \\
\hline MVB spread (SD) & 4.54 & 0.0126 & -2.86 & 0.0487 & 0.00466 & -1.10 & - & 0.271 \\
\hline PFC boost & - & - & -1.45 & - & & -0.132 & - & \\
\hline \multicolumn{9}{|l|}{ SFG } \\
\hline Mean univariate SM & 1.72 & 0.184 & 1.33 & - & 0.181 & 1.34 & - & 0.179 \\
\hline
\end{tabular}

The table lists mean univariate SM effects, the spread (SD) of multivariate Bayesian (MVB) voxel weights predicting SM, and results of the between-region tests of "boost" to model evidence for PFC plus PVC models compared with PVC-only. See text for details. Alpha $=0.0125$. SM, Subsequent memory. $n=119$.

$t_{(118)}=5.91, p<0.0001, r_{\mathrm{adj}}^{2}=0.215$; quadratic effect of age, $t_{(118)}=1.72, p=0.0881$; for PFC, linear effect of age, $t_{(118)}=$ 5.64, $p<0.0001, r_{\mathrm{adj}}^{2}=0.199$; quadratic $t_{(118)}=-2.31, p=$ $\left.0.0226, r_{\text {adj }}^{2}=0.0268\right)$.

To provide a more direct test for a compensatory posteriorto-anterior shift, we assessed the specific contribution of PFC to subsequent memory over and above that of PVC. We fitted a joint MVB model that included both posterior and anterior ROIs and contrasted this with a model including PVC only using Bayesian model comparison. Therefore, we could ask, for each participant, whether adding PFC to the model "boosted" prediction of subsequent memory (see Materials and Methods). Contrary to the PASA theory of a compensatory shift toward greater reliance on PFC, a Bayes factor comparing these two models revealed strong evidence for the null hypothesis of no boost $\left(\mathrm{BF}_{01}=11.1\right)$; indeed, the probability of a boost to model evidence for PVC-PFC compared with PVC-only actually decreased with age numerically (Fig. $3 a$; linear $t_{(118)}=-1.54, p=0.126$ ). Excluding item misses from the model enhanced this pattern (for linear age effect $\left.t_{(107)}=-2.34, p=0.0211, \mathrm{BF}_{01}=14.3\right)$.
Testing subregions within PFC

We also explored whether the pattern of results was similar across subregions within PFC. The PASA theory does not specify which areas are involved in a compensatory shift, but aging does not affect all subregions equally (Raz and Rodrigue, 2006). In functional studies, univariate SM effects in ventrolateral and dorsolateral PFC tend to be age invariant, whereas anterior and superior prefrontal regions show age-related increases (Morcom et al., 2003; Maillet and Rajah, 2014). In contrast, Davis et al.'s (2008) PASA proposal was based on increased activation in older people in anterior ventrolateral PFC and anterior cingulate during visual perception and episodic retrieval. In the present episodic encoding task, there were significant age-related increases in univariate activation in anterior PFC (BA10) and lateral IFG and significant decreases in the spread of multivariate voxel weights in BA10 and medial SFG, as well as numerical decreases in IFG and in lateral middle frontal gyrus including dorsolateral PFC (MFG) (Table 3; see Materials and Methods, "ROI” section, for region definition). Therefore, the overall age-related increase in activation was mainly driven by BA10 and IFG, but no subre- 
Table 4. Age effects on mean univariate SM effects and spread of multivariate SM effects in the STM task

\begin{tabular}{|c|c|c|c|c|c|c|c|c|}
\hline \multirow[b]{2}{*}{$\mathrm{ROI} /$ measure } & \multicolumn{2}{|l|}{ Model } & \multicolumn{3}{|l|}{ Linear } & \multicolumn{3}{|l|}{ Quadratic } \\
\hline & $F$ & $p$ & $t$ & $r_{\text {adj }}^{2}$ & $p$ & $T$ & $r_{\text {adj }}^{2}$ & $p$ \\
\hline \multicolumn{9}{|c|}{ Mean univariate STM activation } \\
\hline PVC & 9.43 & 0.000187 & 4.28 & 0.119 & $<0.0001$ & -0.988 & - & 0.324 \\
\hline PFC-PVC & 0.606 & 0.548 & -0.587 & - & 0.559 & -0.878 & - & 0.380 \\
\hline \multicolumn{9}{|c|}{ Multivariate spread (SD) of STM activity } \\
\hline PFC & 13.4 & $<0.0001$ & 0.662 & - & 0.507 & -5.03 & 0.162 & $<0.0001$ \\
\hline
\end{tabular}

PFC-PVC refers to analyses in which the dependent variable was the difference in each measure between PFC and PVC. $n=96$.

gion showed a decrease in activation with age. The reduction in multivariate information and evidence against a functional boost were relatively uniform over subregions (Table 3). Direct model comparison showed no evidence that PFC activity was compensatory in older age, even in the two subregions with strong increases in activation: Bayes factors weighed against any boost to prediction of subsequent memory for joint PFC-PVC models relative to $\mathrm{PVC}$-only models $\left(\mathrm{BF}_{01}\right.$ favoring the null over positive linear effect of age = 11.1 for BA10, 12.5 for MFG, 5.00 for IFG, and 14.2 for SFG).

\section{Testing posterior-to-anterior shift}

The foregoing analyses provide strong evidence that the increase in (univariate) PFC activity observed in this task did not reflect compensation. Nonetheless, the PASA theory is more general, describing a shift in relative reliance on posterior and anterior regions with age, which need not be compensatory, but could reflect differential age effects in posterior and anterior cortices. In other words, the relative involvement of anterior versus posterior regions could increase with age even if the absolute involvement of both decreased with age. Direct comparison of the mean univariate activation between ROIs did not reveal any evidence for such relative differences in age effects, with strong Bayesian evidence against the predicted greater age-related increase in PFC $\left(\mathrm{BF}_{01}\right.$ for null hypothesis $=25$; Table 2$)$. We next tested for a shift in the relative multivariate information between regions. In the separate MVB models, the age-related reduction in spread of weights was numerically greater in PFC than PVC (linear age effect on PFC-PVC difference, $t_{(118)}=4.16, p=0.0437$; Table 2). We also measured the proportion of top-weighted voxels $(>2$ SDs above the mean) that were located in PFC as opposed to PVC in the joint PVC-PFC model. This proportion decreased significantly with age (overall model, $F_{(2,116)}=3.27, p=0.0415$; linear $t_{(118)}=-2.55, r_{\mathrm{adj}}^{2}=0.359, p=0.0119 ;$ quadratic $t_{(118)}=$ $-0.106, p=0.915)$, with mean $52.4 \%$ of top voxels located in PFC in the younger tertile ( $\mathrm{SD}=9.09 ; 18-45$ years) and $47.1 \%$ in the older tertile ( $\mathrm{SD}=8.57 ; 65-88$ years), although this was no longer significant when item misses were excluded (overall model, $F_{(2,105)}=2.60, p=0.0799$, linear $t_{(107)}=-1.86, p=$ $0.0638)$. Therefore, there was no evidence that, in older age, there is a general shift in the areas contributing to subsequent memory from posterior to anterior (but see Experiment 2 below).

\section{Experiment 2: STM Maintenance}

Behavioral results

For the visual STM task, analysis of the effects of increasing load on performance showed a strong age-related increase in the effect of load on accuracy measured using the root mean squared error of the estimated dot direction relative to the actual dot direction in degrees (Fig. 1b). As expected, older people showed a larger increase in error at load $=3$ compared with load $=1$ (linear contrast) than younger people (for linear age-by-load interaction, $t_{(95)}=5.53, p<0.0001, r_{\mathrm{adj}}^{2}=0.192$; quadratic $t_{(95)}=1.27$, $p=0.203)$, although some age-related decrement in accuracy was present even at load $=1$ (for linear effect of age, $t_{(95)}=2.607, p=$ $0110, r_{\text {adj }}^{2}=0.0382 ;$ quadratic $\left.t_{(95)}=0.388, p=0.699\right)$.

\section{Testing compensation}

For STM, standard univariate activation analyses showed that increasing load elicited activity increases during the maintenance period, which varied according to age in both ROIs. As in the LTM experiment and consistent with the PASA account, PFC activation increased with age (linear $t_{(95)}=3.01, p=0.003$; quadratic $t_{(95)}=-0.505, p=0.615$ ) (Fig. 2c, Table 4). Unlike for LTM encoding, there was also a significant increase in loadrelated PVC activation over the lifespan (linear $t_{(95)}=4.28, p<$ 0.0001 ; quadratic $t_{(93)}=-0.988, p=0.324$; Table 4).

Separate MVB analysis in each ROI showed a similar pattern of age effects to the LTM task. In both PFC and PFC, the spread (SD) of individual voxel weights predicting increased STM load was particularly reduced during later life, with a significant quadratic component (PVC: linear $t_{(95)}=-1.01, p=0.308$; quadratic $t_{(95)}=-4.07, p<0.0001$; PFC: linear $t_{(95)}=0.662, p=$ 0.507; quadratic $t_{(95)}=-5.03, p<0.0001$ ) (Fig. $2 d$, Table 4 ). The result for PVC was unchanged by removing a subset of subjects with very low values (i.e., SD weights $<0.0005$; for quadratic age effect $\left.t_{(69)}=-5.32, p=0.0012\right)$. As found for LTM encoding, the direction of the effect in PFC was contrary to a compensatory PASA shift; that is, PFC voxels contributed less to the cognitive task in old age. Again, the spread of univariate effects did not show the same effects of age (in PVC linear $t_{(95)}=1.52, p=0.134$; quadratic $t_{(95)}=0.831, p=0.406$; in PFC, linear $t_{(95)}=-0.471$, $p=0.641$; quadratic $\left.t_{(95)}=1.70, p=0.0912\right)$.

As for LTM encoding, we used model comparison of a joint PVC-PFC model with a PVC-only model to directly evaluate the compensatory PASA hypothesis. The results were similar to the LTM experiment: The "boost" to prediction of the cognitive variable obtained by adding PFC to the model showed a significant age-related reduction in the probability of a boost for STM load (in an ordinal regression, $t_{(95)}=-2.00, p=0.0479$ ). The Bayes factor provided strong evidence against the compensatory hypothesis of an increased boost (for unidirectional hypothesis, $\mathrm{BF}_{01}=10.2$ ), although evidence was only anecdotal for the presence of an age-related reduction in boost (for bidirectional hypothesis, $\mathrm{BF}_{01}=1.81$ ). Therefore, like for the LTM experiment, there was clear evidence against a compensatory increase in prefrontal contribution to the task with age. 
Table 5. Age effects for PFC subregions in the visual short-term memory task

\begin{tabular}{|c|c|c|c|c|c|c|c|c|}
\hline \multirow[b]{2}{*}{ ROI/ measure } & \multicolumn{2}{|l|}{ Model } & \multicolumn{3}{|l|}{ Linear } & \multicolumn{3}{|l|}{ Quadratic } \\
\hline & $F$ & $P$ & $t$ & $r_{\mathrm{adj}}^{2}$ & $p$ & $t$ & $r_{\text {adj }}^{2}$ & $p$ \\
\hline \multicolumn{9}{|l|}{ BA10 } \\
\hline Mean univariate & 1.90 & 0.155 & 1.79 & - & 0.0787 & -0.937 & - & 0.352 \\
\hline MVB spread (SD) & 12.7 & $<0.0001$ & -1.41 & - & 0.158 & -4.69 & 0.171 & $<0.0001$ \\
\hline PFC boost $t$ & - & - & -1.48 & - & 0.142 & -1.00 & - & 0.320 \\
\hline PFC boost BF 01 & - & - & 10.0 & - & - & - & - & - \\
\hline \multicolumn{9}{|l|}{ IFG } \\
\hline Mean univariate & 2.64 & 0.0767 & 1.99 & - & 0.0512 & 0.997 & - & 0.323 \\
\hline MVB spread (SD) & 6.26 & 0.00282 & -0.787 & - & 0.427 & -3.35 & 0.0864 & 0.00109 \\
\hline PFC boost $t$ & - & - & -1.11 & - & 0.270 & -1.10 & - & 0.274 \\
\hline PFC boost $\mathrm{BF}_{01}$ & - & - & 7.69 & - & - & - & - & - \\
\hline \multicolumn{9}{|l|}{ MFG } \\
\hline Mean univariate & 2.08 & 0.131 & 2.03 & - & 0.0459 & -0.447 & - & 0.654 \\
\hline MVB spread (SD) & 11.0 & $<0.0001$ & 0.300 & - & 0.762 & -4.60 & 0.165 & $<0.0001$ \\
\hline PFC boost $t$ & - & - & -1.38 & - & 0.171 & -0.788 & - & 0.433 \\
\hline PFC boost $\mathrm{BF}_{01}$ & - & - & 6.25 & - & 一 & - & - & - \\
\hline \multicolumn{9}{|l|}{ SFG } \\
\hline Mean univariate & 2.64 & 0.0770 & 2.27 & - & 0.0264 & 0.241 & - & 0.812 \\
\hline MVB spread (SD) & 7.37 & 0.00106 & -1.57 & - & 0.116 & -3.34 & 0.0858 & 0.00111 \\
\hline PFC boost $t$ & - & 一 & -2.73 & 0.0528 & 0.00755 & -0.720 & - & 0.473 \\
\hline PFC boost $\mathrm{BF}_{01}$ & - & - & 14.3 & - & - & - & - & - \\
\hline
\end{tabular}

The table lists mean univariate activation during maintenance in response to increasing VSTM load, the spread (SD) of MVB voxel weights predicting linearly increasing VSTM load, and results of the between-region tests of "boost" to mode evidence for PFC plus PVC models compared with PVC-only. See text for details. Alpha $=0.0125 . n=96$.

Testing subregions within PFC

Again, we examined the four prefrontal subregions separately to assess whether the findings were driven by a specific part or parts of the large ROI (Table 5). For this experiment, the age-related increase in univariate activation was not separately significant in any subregion, which may have reflected relatively distributed effects and the more inclusive selection of "active" voxels. As for the LTM experiment, however, overall age effects on the spread of multivariate voxel weights were significant in three subregions and those in IFG were similar in magnitude and form, suggesting reductions in spread across PFC with no major betweensubregion differences. Moreover, all ROIs showed Bayes factors of at least 6 against a boost to model evidence from adding PFC to the posterior-only models predicting increasing STM load, again consistent with the overall results.

\section{Testing posterior-to-anterior shift}

Finally, even if age increased activity and decreased multivariate information in both PFC and PVC, it is possible that the PFC: $\mathrm{PVC}$ ratio of activity and/or multivariate information increases with age, consistent with the general PASA claim. As for LTM encoding, there was no evidence that age effects on (univariate) activation in the two ROIs differed; that is, the increase in activation in older people was similar in magnitude $\left(\mathrm{BF}_{01}\right.$ for null hypothesis over prediction of a greater age-related increase in PFC $=33.3$; Table 4). However, multivariate analysis revealed a picture different from that in the LTM task. In the separate MVB models, the age-related reduction in spread of weights showed a stronger quadratic component in PFC than PVC (for PFC-PFC, quadratic $t_{(95)}=2.26, p=0.0244$; Table 4). More clearly, when examining the location of top-weighted voxels from the joint PFC + PVC model, a higher proportion were located in PFC in older age (for model, $F_{(2,93)}=22.4, p<0.0001$, linear $t_{(95)}=3.72$, $p<0.001$, quadratic $t_{(95)}=5.20, p<0.0001$ ), with mean $69.1 \%$ of top voxels located in PFC in the younger tertile $(\mathrm{SD}=14.1$; $25-43$ years), but $81.0 \%$ in the older tertile $(\mathrm{SD}=13.3 ; 66-85$ years). Therefore, although the STM experiment, like the LTM experiment, found decreases in absolute PFC (and PVC) involvement in old age, the relative involvement of PFC versus PVC voxels (at least in terms of those with high weights in the joint model) did increase with age, unlike in the LTM experiment. This provides some support for a PASA pattern in this task, even though there was no evidence that this greater PFC involvement was compensatory.

\section{Discussion}

This study investigated the proposal that there is a posterior-toanterior shift in task-related brain activity during aging, with the greater reliance on PFC in older age reflecting compensatory mechanisms. We tested the predictions of this PASA theory with data from two memory tasks that were conducted on independent and relatively large population-derived adult lifespan samples. Using novel model-based multivariate analyses, we provide direct evidence against a compensatory posterior-to-anterior shift. Instead, our data suggest that the increased prefrontal activation reflects less specific or less efficient activity, rather than compensation.

The results of our standard univariate activation analyses are consistent with previous studies showing age-related increases in activation in PFC, which form the basis of the PASA theory (Grady et al., 1994; Davis et al., 2008). Many studies have found such increases in PFC activation in different cognitive tasks, although regional reductions in activation are also found (e.g., see Rajah and D'Esposito, 2005; Spreng et al., 2010; Li et al., 2015). We found such age-related increases in both the PFC activation associated with trials that were later remembered many minutes later (in the LTM experiment) and the activation associated with maintaining increasing numbers of items for a few seconds (in the STM experiment). We also further generalized previous findings across PFC subregions, in that the increased activation was reliable across lateral, anterior, and superior prefrontal areas (although in the LTM experiment, it was mainly driven by inferolateral and anterior PFC).

Importantly, despite this increased univariate activity, multivariate analysis of both experiments showed that with increasing age, $\mathrm{PFC}$ possessed less, rather than more, information about the cognitive outcome. This reduced pattern information was evi- 
dent both in terms of the spread of voxel weights (Fig. 2) and the lack of a meaningful boost to model evidence when adding PFC voxels to the model (Fig. 3). The latter type of inference was made possible by our novel use of MVB classification.

If the increased prefrontal activation with age is not compensatory, then what does it reflect? One possibility is that neural function is less efficient, such that a greater BOLD signal is required for the same level of computation; that is, less "bang for the buck" for the same level of neural activity (see also Rypma and D’Esposito, 2000; Morcom et al., 2007; Grady, 2008; ReuterLorenz and Campbell, 2008; Nyberg et al., 2014). This could reflect the proposed greater sensitivity of prefrontal cortices than other brain regions to aging (West, 1996; Glisky et al., 2001; Raz and Rodrigue, 2006). Another possibility is that PFC activity becomes less specific with age, as might be expected by theories of age-related dedifferentiation, particularly in complex cognitive functions (Li et al., 2001; Park et al., 2004; Carp et al., 2011; Abdulrahman et al., 2017). Partial support for the latter comes from the LTM experiment, where the negative effect of age on the spread of multivariate weights across voxels was accompanied by a positive effect of age on the spread (as well as mean) of univariate activity. This suggests that, although more voxels showed substantial (positive or negative) activity related to subsequent memory in older people, these additional responses were redundant, with fewer voxels contributing uniquely to memory encoding, as expected if the increased prefrontal activity is less specific. Conversely, in the STM experiment, the spread of univariate responses was age invariant, suggesting a more spatially uniform increase in response to load with age, although the MVB results suggested that, just as in the LTM task, this increased response carried less information. Whether the present results reflect reductions in efficiency or reductions in specificity, they are more consistent with the general idea of brain maintenance (Nyberg et al., 2014), that cognitive function in older age is determined by the ability to maintain a youth-like brain, than with the idea associated with PASA of functional compensation by anterior brain regions.

Despite age-related decreases in overall multivariate information in both PFC and PVC, it is possible that the relative contribution of anterior regions to cognitive tasks could increase with age. There is some evidence for such a shift from studies showing crossover effects in which age-related decreases in posterior cortical activity occur alongside age-related increases in PFC (Grady et al., 1994; Davis et al., 2008; see also recent meta-analysis by Maillet and Rajah, 2014). However, our univariate activation analyses showed little evidence of such a relative posterior-toanterior shift: despite increased prefrontal activation, age effects on univariate activation in PFC and PVC did not differ significantly in either experiment. In terms of multivariate information, the LTM experiment actually showed, if anything, a decrease rather than increase in the contribution of PFC relative to PVC. The only comparison that provided some support for a relative increase in anterior contribution was for multivariate information about load in the STM experiment. Therefore, the direction of any relative shift in reliance on PFC versus PVC with age seems to be task-dependent, as opposed to the task-general posteriorto-anterior shift claimed by PASA (Davis et al., 2008; see also Ford and Kensinger, 2017). This is consistent with other metaanalyses finding age-related decreases as well as increases in activation, depending on the task (Spreng et al., 2010; Li et al., 2015). Moreover, most studies have not made the direct statistical comparisons needed to test for anterior-posterior differences in the absence of crossover effects (Morcom and Johnson, 2015). A strength of our approach is that our analyses encompassed large ROIs in both anterior and posterior cortices, as well as direct comparisons between the two.

In summary, our data replicate an increase in PFC activity over the adult lifespan, but do not support the idea that this reflects a compensatory posterior-to-anterior shift, at least in the context of the two memory tasks considered here. Our results are inconsistent both with the proposal that the increased activity is compensatory and with a generalized shift with age to greater relative reliance on PFC. The data are most parsimoniously explained by reduced efficiency or specificity of neural responses, reflecting primary age-related deleterious changes in posterior as well as PFC, which vary in their relative magnitudes according to the task. Our results therefore help to adjudicate between competing accounts of neurocognitive aging while also illustrating the more general ability of MVB to compare models that comprise different sets of voxels, thereby offering an exciting new general way to test the relative contributions of brain regions to cognitive outcomes.

\section{References}

Abdulrahman H, Fletcher PC, Bullmore E, Morcom AM (2017) Dopamine and memory dedifferentiation in aging. Neuroimage 153:211-220. CrossRef Medline

Ashburner J (2007) A fast diffeomorphic image registration algorithm. Neuroimage 38:95-113. CrossRef Medline

Ashburner J, Friston KJ (2005) Unified segmentation. Neuroimage 26:839851. CrossRef Medline

Cabeza R, Daselaar SM, Dolcos F, Prince SE, Budde M, Nyberg L (2004) Task-independent and task-specific age effects on brain activity during working memory, visual attention and episodic retrieval. Cereb Cortex 14:364-375. CrossRef Medline

Cappell KA, Gmeindl L, Reuter-Lorenz PA (2010) Age differences in prefontal recruitment during verbal working memory maintenance depend on memory load. Cortex 46:462-473. CrossRef Medline

Carp J, Park J, Polk TA, Park DC (2011) Age differences in neural distinctiveness revealed by multi-voxel pattern analysis. Neuroimage 56:736743. CrossRef Medline

Chadwick MJ, Bonnici HM, Maguire EA (2012) Decoding information in the human hippocampus: a user's guide. Neuropsychologia 50:31073121. CrossRef Medline

Chadwick MJ, Bonnici HM, Maguire EA (2014) CA3 size predicts the precision of memory recall. Proc Natl Acad Sci U S A 111:10720-10725. CrossRef Medline

Davis SW, Dennis NA, Daselaar SM, Fleck MS, Cabeza R (2008) Que PASA? The posterior-anterior shift in aging. Cereb Cortex 18:1201-1209. CrossRef Medline

Dennis NA, Hayes SM, Prince SE, Madden DJ, Huettel SA, Cabeza R (2008) Effects of aging on the neural correlates of successful item and source memory encoding. J Exp Psychol Learn Mem Cogn 34:791-808. CrossRef Medline

Dienes Z (2014) Using Bayes to get the most out of non-significant results. Front Psychol 5:781. CrossRef Medline

Emrich SM, Riggall AC, Larocque JJ, Postle BR (2013) Distributed patterns of activity in sensory cortex reflect the precision of multiple items maintained in visual short-term memory. J Neurosci 33:6516-6523. CrossRef Medline

Ford JH, Kensinger EA (2017) Age-related reversals in neural recruitment across memory retrieval phases. J Neurosci 37:5172-5182. CrossRef Medline

Friston K, Chu C, Mourão-Miranda J, Hulme O, Rees G, Penny W, Ashburner J (2008) Bayesian decoding of brain images. Neuroimage 39: 181-205. CrossRef Medline

Friston K, Mattout J, Trujillo-Barreto N, Ashburner J, Penny W (2007) Variational free energy and the Laplace approximation. Neuroimage 34: 220-234. CrossRef Medline

Glisky EL, Rubin SR, Davidson PSR (2001) Source memory in older adults: an encoding or retrieval problem? J Exp Psychol Learn Mem Cogn 27: 1131-1146. Medline 
Grady C (2012) The cognitive neuroscience of ageing. Nat Rev Neurosci 13:491-505. CrossRef Medline

Grady CL (2008) Cognitive neuroscience of aging. Ann N Y Acad Sci 1124: 127-144. CrossRef Medline

Grady CL, Maisog JM, Horwitz B, Ungerleider LG, Mentis MJ, Salerno JA, Pietrini P, Wagner E, Haxby JV (1994) Age-related changes in cortical blood flow activation during visual processing of faces and location. J Neurosci 14:1450-1462. CrossRef Medline

Grady CL, McIntosh AR, Bookstein F, Horwitz B, Rapoport SI, Haxby JV (1998) Age-related changes in regional cerebral blood flow during working memory for faces. Neuroimage 8:409-425. CrossRef Medline

Henson RN, Campbell KL, Davis SW, Taylor JR, Emery T, Erzinclioglu S, Erzinclioglu S, Kievit RA (2016) Multiple determinants of lifespan memory differences. Sci Rep 6:32527. CrossRef Medline

Hulme OJ, Skov M, Chadwick MJ, Siebner HR, Ramsøy TZ (2014) Sparse encoding of automatic visual association in hippocampal networks. Neuroimage 102:458-464. CrossRef Medline

JASP Team, 2018. JASP Version 0.8.3.1 [Computer software].

Lancaster JL, Woldorff MG, Parsons LM, Liotti M, Freitas CS, Rainey L, Kochunov PV, Nickerson D, Mikiten SA, Fox PT (2000) Automated talairach atlas labels for functional brain mapping. Hum Brain Mapp 10:120-131. CrossRef Medline

Lang PJ, Bradley MM, Cuthbert BN (1997) International Affective Picture System (IAPS): technical manual and affective ratings. (Tech. Rep. A-6). Gainesville, FL: University of Florida, NIMH Center for the Study of Emotion and Attention.

Li HJ, Hou XH, Liu HH, Yue CL, Lu GM, Zuo XN (2015) Putting agerelated task activation into large-scale brain networks: a meta-analysis of $114 \mathrm{fMRI}$ studies on healthy aging. Neurosci Biobehav Rev 57:156-174. CrossRef Medline

Li SC, Lindenberger U, Sikström S (2001) Aging cognition: From neuromodulation to representation. Trends Cogn Sci 5:479-486. CrossRef Medline

Maass A, Schütze H, Speck O, Yonelinas A, Tempelmann C, Heinze HJ, Berron D, Cardenas-Blanco A, Brodersen KH, Enno Stephan KE, Düzel E (2014) Laminar activity in the hippocampus and entorhinal cortex related to novelty and episodic encoding. Nat Commun 5:5547. CrossRef Medline

Maillet D, Rajah MN (2014) Age-related differences in brain activity in the subsequent memory paradigm: a meta-analysis. Neurosci Biobehav Rev 45:246-257. CrossRef Medline

Maldjian JA, Laurienti PJ, Kraft RA, Burdette JH (2003) An automated method for neuroanatomic and cytoarchitectonic atlas-based interrogation of fMRI data sets. Neuroimage 19:1233-1239. CrossRef Medline

Mattson JT, Wang TH, De Chastelaine M, Rugg MD (2014) Effects of age on negative subsequent memory effects associated with the encoding of item and item-context information. Cereb Cortex 24:3322-3333. CrossRef Medline

Morcom AM, Friston KJ (2012) Decoding episodic memory in ageing: a Bayesian analysis of activity patterns predicting memory. Neuroimage 59:1772-1782. CrossRef Medline

Morcom AM, Johnson W (2015) Neural reorganization and compensation in aging. J Cogn Neurosci 27:1275-1285. CrossRef Medline

Morcom AM, Good CD, Frackowiak RS, Rugg MD (2003) Age effects on the neural correlates of successful memory encoding. Brain 126:213-229. CrossRef Medline

Morcom AM, Li J, Rugg MD (2007) Age effects on the neural correlates of episodic retrieval: Increased cortical recruitment with matched performance. Cereb Cortex 17:2491-2506. CrossRef Medline

Nyberg L, Lövdén M, Riklund K, Lindenberger U, Bäckman L (2012) Memory aging and brain maintenance. Trends Cogn Sci 16:292-305. CrossRef Medline

Nyberg L, Andersson M, Kauppi K, Lundquist A, Persson J, Pudas S, Nilsson LG (2014) Age-related and genetic modulation of frontal cortex efficiency. J Cogn Neurosci 26:746-754. CrossRef Medline

Park DC, Reuter-Lorenz P (2009) The adaptive brain: aging and neurocognitive scaffolding. Annu Rev Psychol 60:173-196. CrossRef Medline

Park DC, Polk TA, Park R, Minear M, Savage A, Smith MR (2004) Aging reduces neural specialization in ventral visual cortex. Proc Natl Acad Sci U S A 101:13091-13095. CrossRef Medline

Rajah MN, D'Esposito M (2005) Region-specific changes in prefrontal function with age: a review of PET and fMRI studies on working and episodic memory. Brain 128:1964-1983. CrossRef Medline

Raz N, Rodrigue KM (2006) Differential aging of the brain: patterns, cognitive correlates and modifiers. Neurosci Biobehav Rev 30:730-748. CrossRef Medline

Reuter-Lorenz PA, Campbell KA (2008) Neurocognitive ageing and the compensation hypothesis. Curr Dir Psychol Sci 17:177-182. CrossRef

Rouder JN, Speckman PL, Sun D, Morey RD, Iverson G (2009) Bayesian $t$ tests for accepting and rejecting the null hypothesis. Psychon Bull Rev 16:225-237. CrossRef Medline

Rypma B, D’Esposito MD (2000) Isolating the neural mechanisms of agerelated changes in human working memory. Nat Neurosci 3:509-515. CrossRef Medline

Shafto MA, Tyler LK, Dixon M, Taylor JR, Rowe JB, Cusack R, Calder AJ, Marslen-Wilson WD, Duncan J, Dalgleish T, Henson RN, Brayne C, Matthews FE; Cam-CAN (2014) The Cambridge Centre for Ageing and Neuroscience (Cam-CAN) study protocol: a cross-sectional, lifespan, multidisciplinary examination of healthy cognitive ageing. BMC Neurol 14:204. CrossRef Medline

Smith AP, Henson RN, Dolan RJ, Rugg MD (2004) fMRI correlates of the episodic retrieval of emotional contexts. Neuroimage 22:868-878. CrossRef Medline

Spreng RN, Wojtowicz M, Grady CL (2010) Reliable differences in brain activity between young and old adults: a quantitative meta-analysis across multiple cognitive domains. Neurosci Biobehav Rev 34:1178-1194. CrossRef Medline

Taylor JR, Williams N, Cusack R, Auer T, Shafto MA, Dixon M, Tyler LK, Cam-CAN, Henson RN (2017) The Cambridge Centre for Ageing and Neuroscience (Cam-CAN) data repository: structural and functional MRI, MEG, and cognitive data from a cross-sectional adult lifespan sample. Neuroimage 144:262-269. CrossRef Medline

Tzourio-Mazoyer N, Landeau B, Papathanassiou D, Crivello F, Etard O, Delcroix N, Mazoyer B, Joliot M (2002) Automated anatomical labeling of activations in SPM using a macroscopic anatomical parcellation of the MNI MRI single-subject brain. Neuroimage 15:273-289. CrossRef Medline

Venables WN, Ripley BD (2002). Modern applied statistics with S. New York, NY: Springer.

Wagenmakers EJ (2007) A practical solution to the pervasive problems of p values. Psychon Bull Rev 14:779-804. CrossRef Medline

West RL (1996) An application of prefrontal cortex function theory to cognitive aging. Psychol Bull 120:272-292. CrossRef Medline 